\title{
Direct Evidence for Neutrino Flavor Transformation from Neutral-Current Interactions in the Sudbury Neutrino Observatory
}

Q. R. Ahmad, ${ }^{17}$ R. C. Allen, ${ }^{4}$ T. C. Andersen, ${ }^{6}$ J. D.Anglin, ${ }^{10}$ J. C. Barton, ${ }^{11, *}$ E. W. Beier, ${ }^{12}$ M. Bercovitch, ${ }^{10}$ J. Bigu, ${ }^{7}$ S. D. Biller, ${ }^{11}$ R. A. Black, ${ }^{11}$ I. Blevis, ${ }^{5}$ R. J. Boardman, ${ }^{11}$ J. Boger, ${ }^{3}$ E. Bonvin, ${ }^{14}$ M. G. Boulay, ${ }^{9,14}$ M. G. Bowler, ${ }^{11}$ T. J. Bowles, ${ }^{9}$ S. J. Brice, ${ }^{9,11}$ M. C. Browne, ${ }^{17,9}$ T. V. Bullard, ${ }^{17}$ G. Bühler, ${ }^{4}$ J. Cameron, ${ }^{11}$ Y. D. Chan, ${ }^{8}$ H. H. Chen, ${ }^{4,}$ M. Chen, ${ }^{14}$ X. Chen, ${ }^{8,11}$ B. T. Cleveland, ${ }^{11}$ E. T. H. Clifford, ${ }^{14}$ J. H. M. Cowan, ${ }^{7}$ D. F. Cowen, ${ }^{12}$ G. A. Cox, ${ }^{17}$ X. Dai, ${ }^{11}$ F. Dalnoki-Veress, ${ }^{5}$ W. F. Davidson, ${ }^{10}$ P. J. Doe, ${ }^{17,9,4}$ G. Doucas, ${ }^{11}$ M. R. Dragowsky, ${ }^{9,8}$ C. A. Duba, ${ }^{17}$ F. A. Duncan, ${ }^{14}$ M. Dunford, ${ }^{12}$ J. A. Dunmore, ${ }^{11}$ E. D. Earle,${ }^{14,1}$ S. R. Elliott, ${ }^{17,9}$ H. C. Evans, ${ }^{14}$ G. T. Ewan, ${ }^{14}$ J. Farine, ${ }^{7,5}$ H. Fergani, ${ }^{11}$ A. P. Ferraris, ${ }^{11}$ R. J. Ford, ${ }^{14}$ J. A. Formaggio, ${ }^{17}$ M. M. Fowler, ${ }^{9}$ K. Frame, ${ }^{11}$ E. D. Frank, ${ }^{12}$ W. Frati, ${ }^{12}$ N. Gagnon, ${ }^{11,9,8,17}$ J. V. Germani, ${ }^{17}$ S. Gil, ${ }^{2}$ K. Graham, ${ }^{14}$ D. R. Grant, ${ }^{5}$ R. L. Hahn, ${ }^{3}$ A. L. Hallin, ${ }^{14}$ E. D. Hallman, ${ }^{7}$ A. S. Hamer, ${ }^{9,14}$ A. A. Hamian, ${ }^{17}$ W. B. Handler, ${ }^{14}$ R. U. Haq, ${ }^{7}$ C. K. Hargrove, ${ }^{5}$ P. J. Harvey, ${ }^{14}$ R. Hazama, ${ }^{17}$ K. M. Heeger, ${ }^{17}$ W. J. Heintzelman, ${ }^{12}$ J. Heise, ${ }^{2,9}$ R. L. Helmer, ${ }^{16,2}$ J. D. Hepburn, ${ }^{14}$ H. Heron, ${ }^{11}$ J. Hewett, ${ }^{7}$ A. Hime, ${ }^{9}$ M. Howe, ${ }^{17}$ J. G. Hykawy, ${ }^{7}$ M. C. P. Isaac, ${ }^{8}$ P. Jagam, ${ }^{6}$ N. A. Jelley, ${ }^{11}$ C. Jillings, ${ }^{14}$ G. Jonkmans, ${ }^{7,1}$ K. Kazkaz, ${ }^{17}$ P. T. Keener, ${ }^{12}$ J. R. Klein, ${ }^{12}$ A. B. Knox, ${ }^{11}$ R. J. Komar, ${ }^{2}$ R. Kouzes, ${ }^{13}$ T. Kutter, ${ }^{2}$ C. C. M. Kyba, ${ }^{12}$ J. Law, ${ }^{6}$ I. T. Lawson, ${ }^{6}$ M. Lay, ${ }^{11}$ H. W. Lee, ${ }^{14}$ K. T. Lesko, ${ }^{8}$ J. R. Leslie, ${ }^{14}$ I. Levine ${ }^{5}$ W. Locke, ${ }^{11}$ S. Luoma, ${ }^{7}$ J. Lyon, ${ }^{11}$ S. Majerus, ${ }^{11}$ H. B. Mak, ${ }^{14}$ J. Maneira, ${ }^{14}$ J. Manor, ${ }^{17}$ A. D. Marino, ${ }^{8}$ N. McCauley, ${ }^{12,11}$ A. B. McDonald, ${ }^{14,13}$ D. S. McDonald,${ }^{12}$ K. McFarlane, ${ }^{5}$ G. McGregor, ${ }^{11}$ R. Meijer Drees, ${ }^{17}$ C. Mifflin, ${ }^{5}$ G. G. Miller, ${ }^{9}$ G. Milton, ${ }^{1}$ B. A. Moffat, ${ }^{14}$ M. Moorhead, ${ }^{11}$ C. W. Nally, ${ }^{2}$ M. S. Neubauer, ${ }^{12}$ F. M. Newcomer, ${ }^{12}$ H. S. Ng, ${ }^{2}$ A. J. Noble, ${ }^{16,5}$ E. B. Norman, ${ }^{8}$ V. M. Novikov, ${ }^{5}$ M. O’Neill, ${ }^{5}$ C. E. Okada ${ }^{8}$ R. W. Ollerhead, ${ }^{6}$ M. Omori, ${ }^{11}$ J. L. Orrell, ${ }^{17}$ S. M. Oser, ${ }^{12}$ A. W. P. Poon, ${ }^{8,17,2,9}$ T. J. Radcliffe, ${ }^{14}$ A. Roberge ${ }^{7}$ B. C. Robertson, ${ }^{14}$ R. G. H. Robertson, ${ }^{17,9}$ S. S. E. Rosendahl, ${ }^{8}$ J. K. Rowley, ${ }^{3}$ V. L. Rusu, ${ }^{12}$ E. Saettler, ${ }^{7}$ K. K. Schaffer, ${ }^{17}$ M. H. Schwendener, ${ }^{7}$ A. Schülke, ${ }^{8}$ H. Seifert, ${ }^{7,17,9}$ M. Shatkay, ${ }^{5}$ J. J. Simpson, ${ }^{6}$ C. J. Sims, ${ }^{11}$ D. Sinclair, ${ }^{5,16}$ P. Skensved, ${ }^{14}$ A. R. Smith, ${ }^{8}$ M. W. E. Smith, ${ }^{17}$ T. Spreitzer, ${ }^{12}$ N. Starinsky, ${ }^{5}$ T. D. Steiger, ${ }^{17}$ R. G. Stokstad, ${ }^{8}$ L. C. Stonehill, ${ }^{17}$ R. S. Storey, ${ }^{10}$ B. Sur, ${ }^{1,14}$ R. Tafirout, ${ }^{7}$ N. Tagg, ${ }^{6,11}$ N. W. Tanner, ${ }^{11}$ R. K. Taplin, ${ }^{11}$ M. Thorman, ${ }^{11}$ P. M. Thornewell, ${ }^{11}$ P. T. Trent, ${ }^{11}$ Y. I. Tserkovnyak, ${ }^{2}$ R. Van Berg, ${ }^{12}$ R. G. Van de Water, ${ }^{9,12}$ C. J. Virtue, ${ }^{7}$ C. E. Waltham, ${ }^{2}$ J.-X. Wang, ${ }^{6}$ D. L. Wark, ${ }^{15,11,9}$ N. West, ${ }^{11}$ J. B. Wilhelmy, ${ }^{9}$ J. F. Wilkerson, ${ }^{17,9}$ J. R. Wilson, ${ }^{11}$ P. Wittich, ${ }^{12}$ J. M. Wouters, ${ }^{9}$ and M. Yeh ${ }^{3}$ (SNO Collaboration)

${ }^{1}$ Atomic Energy of Canada, Limited, Chalk River Laboratories, Chalk River, Ontario KOJ 1JO, Canada

${ }^{2}$ Department of Physics and Astronomy, University of British Columbia, Vancouver, British Columbia V6T 1Z1, Canada ${ }^{3}$ Chemistry Department, Brookhaven National Laboratory, Upton, New York 11973-5000

${ }^{4}$ Department of Physics, University of California, Irvine, California 92717 ${ }^{5}$ Carleton University, Ottawa, Ontario K1S 5B6, Canada

${ }^{6}$ Physics Department, University of Guelph, Guelph, Ontario N1G 2W1, Canada

${ }^{7}$ Department of Physics and Astronomy, Laurentian University, Sudbury, Ontario P3E 2C6, Canada

${ }^{8}$ Institute for Nuclear and Particle Astrophysics and Nuclear Science Division, Lawrence Berkeley National Laboratory, Berkeley, California 94720

${ }^{9}$ Los Alamos National Laboratory, Los Alamos, New Mexico 87545

${ }^{10}$ National Research Council of Canada, Ottawa, Ontario K1A OR6, Canada

${ }^{11}$ Department of Physics, University of Oxford, Denys Wilkinson Building, Keble Road, Oxford OX1 3RH, United Kingdom

${ }^{12}$ Department of Physics and Astronomy, University of Pennsylvania, Philadelphia, Pennsylvania 19104-6396

${ }^{13}$ Department of Physics, Princeton University, Princeton, New Jersey 08544

${ }^{14}$ Department of Physics, Queen's University, Kingston, Ontario K7L 3N6, Canada

${ }^{15}$ Rutherford Appleton Laboratory, Chilton, Didcot, Oxon OX11 OQX, United Kingdom

and University of Sussex, Physics and Astronomy Department, Brighton BN1 9QH, United Kingdom

${ }^{16}$ TRIUMF, 4004 Wesbrook Mall, Vancouver, British Columbia V6T 2A3, Canada

${ }^{17}$ Center for Experimental Nuclear Physics and Astrophysics, and Department of Physics, University of Washington, Seattle, Washington 98195

(Received 19 April 2002; published 13 June 2002)

Observations of neutral-current $\nu$ interactions on deuterium in the Sudbury Neutrino Observatory are reported. Using the neutral current (NC), elastic scattering, and charged current reactions and assuming the standard ${ }^{8} \mathrm{~B}$ shape, the $\nu_{e}$ component of the ${ }^{8} \mathrm{~B}$ solar flux is $\phi_{e}=1.76_{-0.05}^{+0.05}$ (stat) $)_{-0.09}^{+0.09}$ (syst) $\times 10^{6} \mathrm{~cm}^{-2} \mathrm{~s}^{-1}$ for a kinetic energy threshold of $5 \mathrm{MeV}$. The non- $\nu_{e}$ component is $\phi_{\mu \tau}=3.41_{-0.45}^{+0.45}(\mathrm{stat})_{-0.45}^{+0.48}(\mathrm{syst}) \times 10^{6} \mathrm{~cm}^{-2} \mathrm{~s}^{-1}, 5.3 \sigma$ greater than zero, providing 
strong evidence for solar $\nu_{e}$ flavor transformation. The total flux measured with the NC reaction is $\phi_{\mathrm{NC}}=5.09_{-0.43}^{+0.44}(\text { stat })_{-0.43}^{+0.46}($ syst $) \times 10^{6} \mathrm{~cm}^{-2} \mathrm{~s}^{-1}$, consistent with solar models.

DOI: 10.1103/PhysRevLett.89.011301

The Sudbury Neutrino Observatory (SNO) detects ${ }^{8} \mathrm{~B}$ solar neutrinos through the reactions:

$$
\begin{aligned}
\nu_{e}+d & \rightarrow p+p+e^{-} \quad(\mathrm{CC}), \\
\nu_{x}+d & \rightarrow p+n+\nu_{x} \quad(\mathrm{NC}), \\
\nu_{x}+e^{-} & \rightarrow \nu_{x}+e^{-} \quad(\mathrm{ES}) .
\end{aligned}
$$

The charged current (CC) reaction is sensitive exclusively to electron-type neutrinos, while the neutral current (NC) reaction is equally sensitive to all active neutrino flavors $(x=e, \mu, \tau)$. The elastic scattering (ES) reaction is sensitive to all flavors as well, but with reduced sensitivity to $\nu_{\mu}$ and $\nu_{\tau}$. Sensitivity to these three reactions allows SNO to determine the electron and nonelectron active neutrino components of the solar flux [1]. The CC and ES reaction results have recently been presented [2]. This Letter presents the first NC results and updated CC and ES results from SNO.

SNO [3] is a water Cherenkov detector located at a depth of $6010 \mathrm{~m}$ of water equivalent in the INCO, Ltd. Creighton mine near Sudbury, Ontario, Canada. The detector uses ultrapure heavy water contained in a transparent acrylic spherical shell $12 \mathrm{~m}$ in diameter to detect solar neutrinos. Cherenkov photons generated in the heavy water are detected by 9456 photomultiplier tubes (PMTs) mounted on a stainless steel geodesic sphere $17.8 \mathrm{~m}$ in diameter. The geodesic sphere is immersed in ultrapure light water to provide shielding from radioactivity in both the PMT array and the cavity rock.

The data reported here were recorded between 2 November 1999 and 28 May 2001 and represent a total of 306.4 live days, spanning the entire first phase of the experiment, in which only $\mathrm{D}_{2} \mathrm{O}$ was present in the sensitive volume. The analysis procedure was similar to that described in [2]. PMT times and hit patterns were used to reconstruct event vertices and directions and to assign to each event a most probable kinetic energy, $T_{\text {eff }}$. The total flux of active ${ }^{8} \mathrm{~B}$ solar neutrinos with energies greater than $2.2 \mathrm{MeV}$ (the $\mathrm{NC}$ reaction threshold) was measured with the NC signal (Cherenkov photons resulting from the $6.25 \mathrm{MeV} \gamma$ ray from neutron capture on deuterium). The analysis threshold was $T_{\text {eff }} \geq 5 \mathrm{MeV}$, providing sensitivity to neutrons from the $\mathrm{NC}$ reaction. Above this energy threshold, there were contributions from $\mathrm{CC}$ events in the $\mathrm{D}_{2} \mathrm{O}$, ES events in the $\mathrm{D}_{2} \mathrm{O}$ and $\mathrm{H}_{2} \mathrm{O}$, capture of neutrons (both from the $\mathrm{NC}$ reaction and backgrounds), and low energy Cherenkov background events.

A fiducial volume was defined to accept only events which had reconstructed vertices within $550 \mathrm{~cm}$ from the detector center to reduce external backgrounds and systematic uncertainties associated with optics and event reconstruction near the acrylic vessel. The neutron response and systematic uncertainty was calibrated with a ${ }^{252} \mathrm{Cf}$

011301-2
PACS numbers: 26.65.+t, 14.60.Pq, 95.85.Ry

source. The deduced efficiency for neutron captures on deuterium is $29.9 \pm 1.1 \%$ for a uniform source of neutrons in the $\mathrm{D}_{2} \mathrm{O}$. The neutron detection efficiency within the fiducial volume and above the energy threshold is $14.4 \%$. The energy calibration was updated from [2] with the ${ }^{16} \mathrm{~N}$ calibration source [4] data and Monte Carlo calculations. The energy response for electrons, updated for the lower analysis threshold, was characterized as a Gaussian function with resolution $\sigma_{T}=-0.0684+0.331 \sqrt{T_{e}}+$ $0.0425 T_{e}$, where $T_{e}$ is the true electron kinetic energy in $\mathrm{MeV}$. The energy scale uncertainty is $1.2 \%$.

The primary backgrounds to the NC signal are due to low levels of uranium and thorium decay chain daughters $\left({ }^{214} \mathrm{Bi}\right.$ and $\left.{ }^{208} \mathrm{Tl}\right)$ in the detector materials. These activities generate free neutrons in the $\mathrm{D}_{2} \mathrm{O}$, from deuteron photodisintegration (pd), and low energy Cherenkov events. Ex situ assays and in situ analysis of the low energy (4-4.5 MeV) Cherenkov signal region provide independent uranium and thorium photodisintegration background measurements.

Two ex situ assay techniques were employed to determine average levels of uranium and thorium in water. Radium ions were directly extracted from the water onto either $\mathrm{MnO}_{x}$ or hydrous Ti oxide (HTiO) ion exchange media. Radon daughters in the $\mathrm{U}$ and $\mathrm{Th}$ chains were subsequently released, identified by $\alpha$ spectroscopy, or the radium was concentrated and the number of decay daughter $\beta-\alpha$ coincidences determined. Typical assays circulated approximately 400 tonnes of water through the extraction media. These techniques provide isotopic identification of the decay daughters and contamination levels in the assayed water volumes, presented in Fig. 1(a). Secular equilibrium in the $U$ decay chain was broken by the ingress of long-lived (3.8 day half-life) ${ }^{222} \mathrm{Rn}$ in the experiment. Measurements of this background were made by periodically extracting and cryogenically concentrating ${ }^{222} \mathrm{Rn}$ from water degassers. Radon from several tonne assays was subsequently counted in $\mathrm{ZnS}(\mathrm{Ag})$ scintillation cells [5]. The radon results are presented [as mass fractions in $\left.g(U) / g\left(\mathrm{D}_{2} \mathrm{O}\right)\right]$ in Fig. 1(b).

Independent measurements of $\mathrm{U}$ and Th decay chains were made by analyzing Cherenkov light produced by the radioactive decays. The $\beta$ and $\beta-\gamma$ decays from the $\mathrm{U}$ and Th chains dominate the low energy monitoring window. Events in this window monitor $\gamma$ rays that produce photodisintegration in these chains $\left(E_{\gamma}>2.2 \mathrm{MeV}\right)$. Cherenkov events fitted within $450 \mathrm{~cm}$ from the detector center and extracted from the neutrino data set provide a time-integrated measure of these backgrounds over the same time period and within the fiducial volume of the neutrino analysis. Statistical separation of in situ $\mathrm{Tl}$ and Bi events was obtained by analyzing the Cherenkov signal isotropy. Tl decays always result in a $\beta$ and a $2.614 \mathrm{MeV}$ $\gamma$, while in this energy window Bi decays are dominated

011301-2 


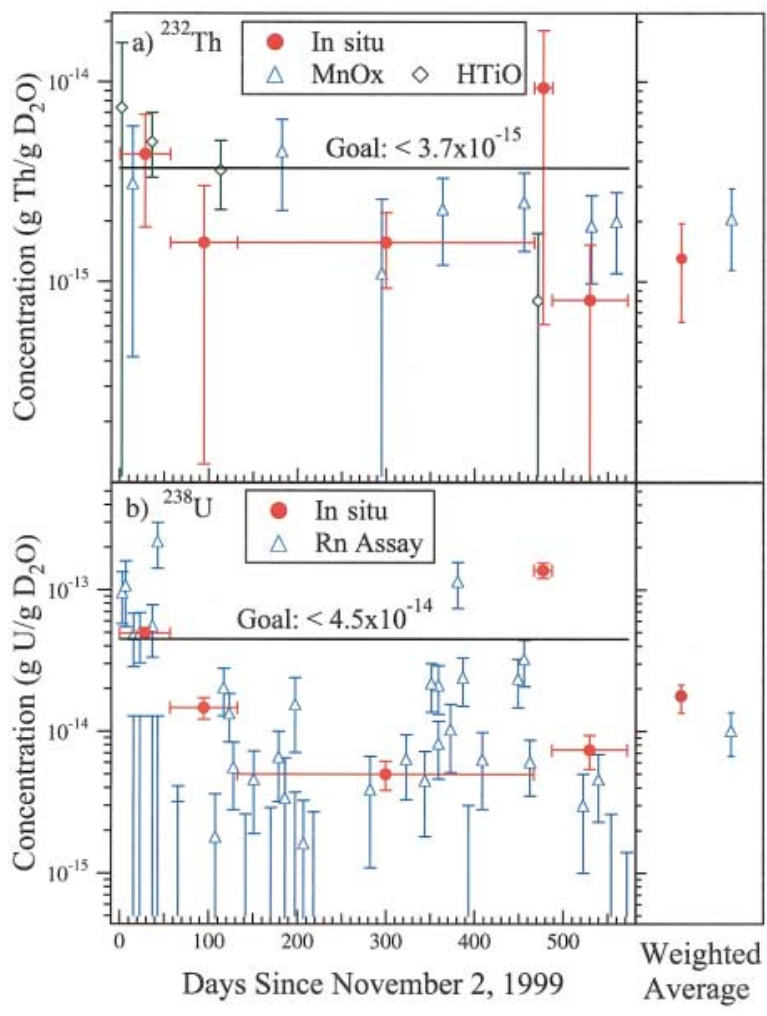

FIG. 1 (color). Thorium (a) and uranium (b) backgrounds (equivalent equilibrium concentrations) in the $\mathrm{D}_{2} \mathrm{O}$ deduced by in situ and ex situ techniques. The $\mathrm{MnO}_{x}$ and HTiO radiochemical assay results, the $\mathrm{Rn}$ assay results, and the in situ Cherenkov signal determination of the backgrounds are presented for the period of this analysis on the left-hand side of frames (a) and (b). The right-hand side shows timeintegrated averages including an additional sampling systematic uncertainty for the ex situ measurement.

by decays with only a $\beta$, and produce, on average, more anisotropic hit patterns.

Results from the ex situ and in situ methods are consistent with each other as shown on the right-hand side of Figs. 1(a) and 1(b). For the ${ }^{232} \mathrm{Th}$ chain, the weighted mean (including additional sampling systematic uncertainty) of the two determinations was used for the analysis. The ${ }^{238} \mathrm{U}$ chain activity is dominated by $\mathrm{Rn}$ ingress which is highly time dependent. Therefore the in situ determination was used for this activity as it provides the appropriate time weighting. The average rate of background neutron production from activities in the $\mathrm{D}_{2} \mathrm{O}$ region is $1.0 \pm 0.2$ neutrons per day, leading to $44_{-9}^{+8}$ detected background events. The production rate from external activities is $1.3_{-0.5}^{+0.4}$ neutrons per day, which leads to $27 \pm 8$ background events since the neutron capture efficiency is reduced for neutrons born near the heavy water boundary. The total photodisintegration background corresponds to approximately $12 \%$ of the number of $\mathrm{NC}$ neutrons predicted by the standard solar model from ${ }^{8} \mathrm{~B}$ neutrinos.

Low energy backgrounds from Cherenkov events in the signal region were evaluated by using acrylic encapsulated sources of $\mathrm{U}$ and Th deployed throughout the detector volume and by Monte Carlo calculations. Probability density functions (pdfs) in reconstructed vertex radius derived from $\mathrm{U}$ and $\mathrm{Th}$ calibration data were used to determine the number of background Cherenkov events from external regions which either entered or misreconstructed into the fiducial volume. Cherenkov event backgrounds from activities in the $\mathrm{D}_{2} \mathrm{O}$ were evaluated with Monte Carlo calculations.

Table I shows the number of photodisintegration and Cherenkov background events (including systematic uncertainties) due to activity in the $\mathrm{D}_{2} \mathrm{O}$ (internal region), acrylic vessel (AV), $\mathrm{H}_{2} \mathrm{O}$ (external region), and PMT array. Other sources of free neutrons in the $\mathrm{D}_{2} \mathrm{O}$ region are cosmic ray events and atmospheric neutrinos. To reduce these backgrounds, an additional neutron background cut imposed a 250-ms dead time (in software) following every event in which the total number of PMTs which registered a hit was greater than 60 . The number of remaining NC atmospheric neutrino events and background events generated by sub-Cherenkov threshold muons is estimated to be small, as shown in Table I.

The data recorded during the pure $\mathrm{D}_{2} \mathrm{O}$ detector phase are shown in Fig. 2. These data have been analyzed using the same data reduction described in [2], with the addition of the new neutron background cut, yielding 2928 events in the energy region selected for analysis, 5 to $20 \mathrm{MeV}$. Figure 2(a) shows the distribution of selected events in the cosine of the angle between the Cherenkov event direction and the direction from the Sun $\left(\cos \theta_{\odot}\right)$ for the analysis threshold of $T_{\text {eff }} \geq 5 \mathrm{MeV}$ and fiducial volume selection of $R \leq 550 \mathrm{~cm}$, where $R$ is the reconstructed event radius. Figure 2(b) shows the distribution of events in the volumeweighted radial variable $\left(R / R_{\mathrm{AV}}\right)^{3}$, where $R_{\mathrm{AV}}=600 \mathrm{~cm}$ is the radius of the acrylic vessel. Figure 2(c) shows the kinetic energy spectrum of the selected events.

In order to test the null hypothesis, the assumption that there are only electron neutrinos in the solar neutrino

TABLE I. Neutron and Cherenkov background events.

\begin{tabular}{lc}
\hline \hline \multicolumn{1}{c}{ Source } & Events \\
\hline $\mathrm{D}_{2} \mathrm{O}$ photodisintegration & $44_{-9}^{+8}$ \\
$\mathrm{H}_{2} \mathrm{O}+\mathrm{AV}$ photodisintegration & $27_{-8}^{+8}$ \\
Atmospheric $\nu$ 's and & $4 \pm 1$ \\
Fission & $\ll 1$ \\
$\quad$ sub-Cherenkov threshold $\mu$ 's & \\
${ }^{2} \mathrm{H}(\alpha, \alpha)$ pn & $2 \pm 0.4$ \\
${ }^{17} \mathrm{O}(\alpha, \mathrm{n})$ & $\ll 1$ \\
Terrestrial and reactor $\bar{\nu}$ 's & $1_{-1}^{+3}$ \\
External neutrons & $\ll 1$ \\
Total neutron background & $78 \pm$ \\
$\mathrm{D}_{2} \mathrm{O}$ Cherenkov & 12 \\
$\mathrm{H}_{2} \mathrm{O}$ Cherenkov & $20_{-6}^{+13}$ \\
$\mathrm{AV}$ Cherenkov & $3_{-3}^{+4}$ \\
PMT Cherenkov & $6_{-6}^{+3}$ \\
Total Cherenkov background & $16_{-8}^{+11}$ \\
\hline \hline
\end{tabular}



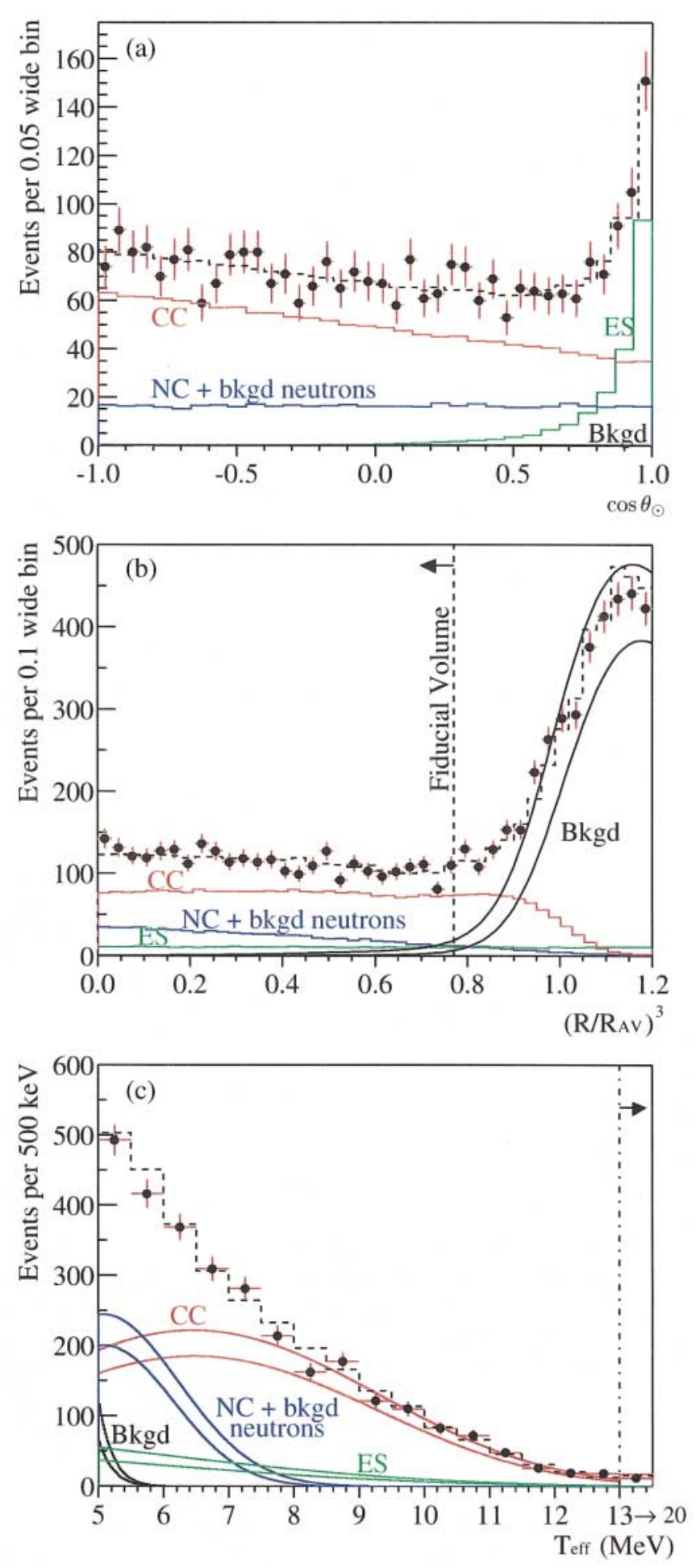

FIG. 2 (color). (a) Distribution of $\cos \theta_{\odot}$ for $R \leq 550 \mathrm{~cm}$. (b) Distribution of the volume weighted radial variable $\left(R / R_{\mathrm{AV}}\right)^{3}$. (c) Kinetic energy for $R \leq 550 \mathrm{~cm}$. Also shown are the Monte Carlo predictions for CC, ES, and NC + bkgd neutron events scaled to the fit results, and the calculated spectrum of Cherenkov background (bkgd) events. The dashed lines represent the summed components, and the bands show $\pm 1 \sigma$ uncertainties. All distributions are for events with $T_{\text {eff }} \geq 5 \mathrm{MeV}$.

flux, the data are resolved into contributions from $\mathrm{CC}$, $\mathrm{ES}$, and NC events above threshold using pdfs in $T_{\text {eff }}$, $\cos \theta_{\odot}$, and $\left(R / R_{\mathrm{AV}}\right)^{3}$, derived from Monte Carlo calculations generated assuming no flavor transformation and the standard ${ }^{8} \mathrm{~B}$ spectral shape [6]. Background event pdfs are included in the analysis with fixed amplitudes determined by the background calibration. The extended maximum likelihood method used in the signal decomposition yields $1967.7_{-60.9}^{+61.9} \mathrm{CC}$ events, 263.6 $6_{-25.6}^{+26.4} \mathrm{ES}$ events, and $576.5_{-48.9}^{+49.5} \mathrm{NC}$ events [7], where only statistical uncertainties are given. Systematic uncertainties on fluxes derived by repeating the signal decomposition with perturbed pdfs (constrained by calibration data) are shown in Table II.

Normalized to the integrated rates above the kinetic energy threshold of $T_{\text {eff }} \geq 5 \mathrm{MeV}$, the flux of ${ }^{8} \mathrm{~B}$ neutrinos measured with each reaction in SNO, assuming the standard spectrum shape [6] is (all fluxes are presented in units of $10^{6} \mathrm{~cm}^{-2} \mathrm{~s}^{-1}$ )

$$
\begin{aligned}
\phi_{\mathrm{CC}}^{\mathrm{SNO}} & =1.76_{-0.05}^{+0.06}(\mathrm{stat})_{-0.09}^{+0.09} \text { (syst)}, \\
\phi_{\mathrm{ES}}^{\mathrm{SNO}} & =2.39_{-0.23}^{+0.24}(\mathrm{stat})_{-0.12}^{+0.12} \text { (syst)}, \\
\phi_{\mathrm{NC}}^{\mathrm{SNO}} & =5.09_{-0.43}^{+0.44}(\mathrm{stat})_{-0.43}^{+0.46} \text { (syst) } .
\end{aligned}
$$

Electron neutrino cross sections are used to calculate all fluxes. The CC and ES results reported here are consistent with the earlier SNO results [2] for $T_{\text {eff }} \geq 6.75 \mathrm{MeV}$. The excess of the NC flux over the CC and ES fluxes implies neutrino flavor transformations.

A simple change of variables resolves the data directly into electron $\left(\phi_{e}\right)$ and nonelectron $\left(\phi_{\mu \tau}\right)$ components [9],

$$
\begin{aligned}
\phi_{e} & =1.76_{-0.05}^{+0.05}(\mathrm{stat})_{-0.09}^{+0.09} \text { (syst) }, \\
\phi_{\mu \tau} & =3.41_{-0.45}^{+0.45}(\text { stat })_{-0.45}^{+0.48} \text { (syst), }
\end{aligned}
$$

assuming the standard ${ }^{8} \mathrm{~B}$ shape. Combining the statistical and systematic uncertainties in quadrature, $\phi_{\mu \tau}$ is $3.41_{-0.64}^{+0.66}$, which is $5.3 \sigma$ above zero, providing strong evidence for flavor transformation consistent with neutrino oscillations [10,11]. Adding the SuperKamiokande ES measurement of the $8 \mathrm{~B}$ flux [12] $\phi_{\mathrm{ES}}^{\mathrm{SK}}=2.32 \pm 0.03$ (stat) ${ }_{-0.07}^{+0.08}$ (syst) as an additional constraint, we find $\phi_{\mu \tau}=3.45_{-0.62}^{+0.65}$, which is $5.5 \sigma$ above zero. Figure 3 shows the flux of nonelectron flavor active neutrinos vs the flux of electron neutrinos deduced from the SNO data. The three bands represent the one standard deviation measurements of the $\mathrm{CC}, \mathrm{ES}$, and $\mathrm{NC}$ rates. The error ellipses represent the $68 \%, 95 \%$, and $99 \%$ joint probability contours for $\phi_{e}$ and $\phi_{\mu \tau}$.

Removing the constraint that the solar neutrino energy spectrum is undistorted, the signal decomposition is repeated using only the $\cos \theta_{\odot}$ and $\left(R / R_{\mathrm{AV}}\right)^{3}$ information. The total flux of active ${ }^{8} \mathrm{~B}$ neutrinos measured with the $\mathrm{NC}$ reaction is

$$
\phi_{\mathrm{NC}}^{\mathrm{SNO}}=6.42_{-1.57}^{+1.57}(\mathrm{stat})_{-0.58}^{+0.55} \text { (syst) }
$$

which is in agreement with the shape constrained value above and with the standard solar model (SSM) prediction [13] for ${ }^{8} \mathrm{~B}, \phi_{\mathrm{SSM}}=5.05_{-0.81}^{+1.01}$. 
TABLE II. Systematic uncertainties on fluxes. The experimental uncertainty for ES (not shown) is $-4.8,+5.0$ percent.

\begin{tabular}{lccc}
\hline \hline \multicolumn{1}{c}{ Source } & $\begin{array}{c}\text { CC uncertainty } \\
\text { (percent) }\end{array}$ & $\begin{array}{c}\text { NC uncertainty } \\
\text { (percent) }\end{array}$ & $\begin{array}{c}\phi_{\mu \tau} \text { uncertainty } \\
\text { (percent) }\end{array}$ \\
\hline Energy scale $^{\mathrm{a}}$ & $-4.2,+4.3$ & $-6.2,+6.1$ & $-10.4,+10.3$ \\
Energy resolution $^{\mathrm{a}}$ & $-0.9,+0.0$ & $-0.0,+4.4$ & $-0.0,+6.8$ \\
Energy nonlinearity $^{\mathrm{a}}$ & \pm 0.1 & \pm 0.4 & \pm 0.6 \\
Vertex resolution $^{\mathrm{a}}$ & \pm 0.0 & \pm 0.1 & \pm 0.2 \\
Vertex accuracy $_{\text {Angular resolution }}$ & $-2.8,+2.9$ & \pm 1.8 & \pm 1.4 \\
Internal source pd $^{\mathrm{a}}$ & $-0.2,+0.2$ & $-0.3,+0.3$ & $-0.3,+0.3$ \\
External source pd $_{\text {DO2 Cherenkov }}^{\mathrm{a}}$ & \pm 0.0 & $-1.5,+1.6$ & $-2.0,+2.2$ \\
HO2 Cherenkov & \pm 0.1 & $-1.0,+1.0$ & \pm 1.4 \\
AV Cherenkov & $-0.1,+0.2$ & $-2.6,+1.2$ & $-3.7,+1.7$ \\
PMT Cherenkov & \pm 0.0 & $-0.2,+0.4$ & $-0.2,+0.6$ \\
Neutron capture & \pm 0.0 & $-0.2,+0.2$ & $-0.3,+0.3$ \\
Cut acceptance & \pm 0.1 & $-2.1,+1.6$ & $-3.0,+2.2$ \\
Experimental uncertainty & \pm 0.0 & $-4.0,+3.6$ & $-5.8,+5.2$ \\
Cross section [8] & $-0.2,+0.4$ & $-0.2,+0.4$ & $-0.2,+0.4$ \\
\hline \hline
\end{tabular}

${ }^{\text {aDenotes }} \mathrm{CC}$ vs NC anticorrelation.

In summary, the results presented here are the first direct measurement of the total flux of active ${ }^{8} \mathrm{~B}$ neutrinos arriving from the Sun and provide strong evidence for neutrino flavor transformation. The $\mathrm{CC}$ and $\mathrm{ES}$ reaction rates are consistent with the earlier results [2] and with the NC reaction rate under the hypothesis of flavor transformation. The total flux of ${ }^{8} \mathrm{~B}$ neutrinos measured with the NC reaction is in agreement with the SSM prediction.

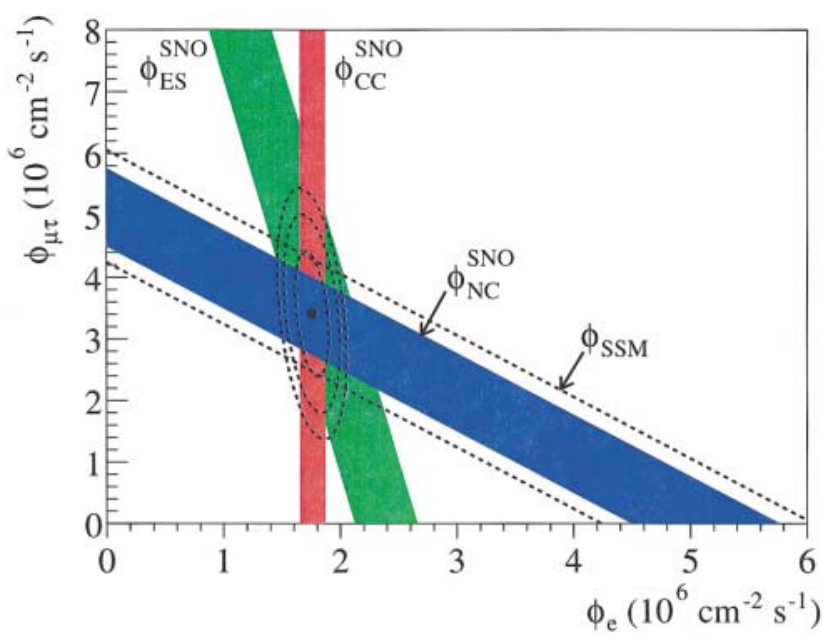

FIG. 3 (color). Flux of ${ }^{8} \mathrm{~B}$ solar neutrinos which are $\mu$ or $\tau$ flavor vs flux of electron neutrinos deduced from the three neutrino reactions in SNO. The diagonal bands show the total ${ }^{8} \mathrm{~B}$ flux as predicted by the SSM [13] (dashed lines) and that measured with the NC reaction in SNO (solid band). The intercepts of these bands with the axes represent the $\pm 1 \sigma$ errors. The bands intersect at the fit values for $\phi_{e}$ and $\phi_{\mu \tau}$, indicating that the combined flux results are consistent with neutrino flavor transformation assuming no distortion in the ${ }^{8} \mathrm{~B}$ neutrino energy spectrum.
This research was supported by Canada: NSERC, Industry Canada, NRC, Northern Ontario Heritage Fund Corporation, Inco, AECL, Ontario Power Generation; U.S.: Department of Energy; U.K.: PPARC. We thank the SNO technical staff for their strong contributions.

*Permanent address: Birkbeck College, University of London, Malet Road, London WC1E 7HX, UK.

${ }^{\dagger}$ Deceased.

[1] H. H. Chen, Phys. Rev. Lett. 55, 1534 (1985).

[2] Q. R. Ahmad et al., Phys. Rev. Lett. 87, 071301 (2001).

[3] SNO Collaboration, J. Boger et al., Nucl. Instrum. Methods Phys. Res., Sect. A 449, 172 (2000).

[4] M. R. Dragowsky et al., Nucl. Instrum. Methods Phys. Res., Sect. A 481, 284 (2002).

[5] M.-Q. Liu, H. W. Lee, and A. B. McDonald, Nucl. Instrum. Methods Phys. Res., Sect. A 329, 291 (1993).

[6] C. E. Ortiz et al., Phys. Rev. Lett. 85, 2909 (2000).

[7] We note that this rate of neutron events also leads to a lower bound on the proton lifetime for "invisible" modes \{based on the free neutron that would be left in deuterium [V. I. Tretyak and Yu. G. Zdesenko, Phys. Lett. B 505, 59 (2001)] in excess of $10^{28}$ years, approximately 3 orders of magnitude more restrictive than previous limits [J. Evans and R. Steinberg, Science 197, 989 (1977)]\}. The possible contribution of this mechanism to the solar neutrino NC background is ignored.

[8] Cross section uncertainty includes $g_{A}$ uncertainty $(0.6 \%)$, difference between NSGK [S. Nakamura, T. Sato, V. Gudkov, and K. Kubodera, Phys. Rev. C 63, 034617 (2001)] and BCK [M. Butler, J.-W. Chen, and X. Kong, Phys. Rev. C 63, 035501 (2001)] in SNO's calculations (0.6\%), radiative correction uncertainties $[0.3 \%$ for $\mathrm{CC}, 0.1 \%$ for NC; A. Kurylov, M. J. Ramsey-Musolf, and P. Vogel, Phys. Rev. C 65, 055501 (2002)], uncertainty associated with neglect of real photons in SNO $(0.7 \%$ for CC), and 
theoretical cross section uncertainty $[1 \%, \mathrm{~S}$. Nakamura et al., arXiv:nucl-th/0201062 (to be published)].

[9] This change of variables allows a direct test of the null hypothesis of no flavor transformation $\left(\phi_{\mu \tau}=0\right)$ without requiring calculation of the $\mathrm{CC}, \mathrm{ES}$, and $\mathrm{NC}$ signal correlations.
[10] Z. Maki, N. Nakagawa, and S. Sakata, Prog. Theor. Phys. 28, 870 (1962).

[11] V. Gribov and B. Pontecorvo, Phys. Lett. 28B, 493 (1969).

[12] S. Fukuda et al., Phys. Rev. Lett. 86, 5651 (2001).

[13] John N. Bahcall, M. H. Pinsonneault, and Sarbani Basu, Astrophys. J. 555, 990 (2001). 